\title{
Tratamiento postoperatorio en el paciente diagnosticado de cáncer de mama
}

\section{Post-operative treatment in breast cancer diagnosed patient}

\author{
G. Paseiro, ${ }^{1}$ M. Mourelle Zas, ${ }^{2}$ C. Veiras Candal, ${ }^{3}$ M.C. Silva Rodríguez, ${ }^{4}$ \\ ${ }^{1}$ Fisioterapeuta. Profesor titular de Escuela Universitaria en la Universidad de A Coruña. Departamento de \\ Fisioterapia \\ ${ }^{2}$ Licenciada en Educación Física y Deporte. Profesora de Educación Secundaria \\ ${ }^{3}$ Licenciado en Medicina. Director Médico del Centro Oncológico Regional de Galicia \\ ${ }^{4}$ Licenciada en Medicina. Jefa Clínica del Departamento de Oncología Radioterápica del Centro Oncológico \\ Regional de Galicia
}

\section{Resumen}

El cáncer, que es la causa más frecuente de muerte prematura y evitable en la Unión Europea, constituye un problema importante de salud pública. Dentro del colectivo femenino, uno de los cánceres más frecuentes y que más preocupan a la población es el cáncer de mama. En Europa se estima que 8 de cada 100 mujeres desarrollarán está enfermedad antes de los 75 años.

La cirugía y la radioterapia pueden provocar alteraciones postquirúrgicas que a la larga disminuyen la calidad de vida de estas pacientes.

Con este artículo se pretende realizar una búsqueda bibliográfica que clarifique las alteraciones postquirúrgicas en este tipo de paciente y el tratamiento fisioterápico que se emplea para tratarlas.

Todos los estudios analizados indican la aparición de alteraciones glenohumerales en estos pacientes y los beneficios que el tratamiento de fisioterapia les acarrea, ningún estudio atestigua resultados contrarios. Si bien es cierto que no existe ningún estudio con la suficiente muestra para aclarar que tipo de pacientes (edad, condición física, tipo de cirugía, radioterapia recibida) presentan una incidencia mayor de estas alteraciones.

\begin{abstract}
Cancer is the most frequent cause of premature and avoidable death in the European Union, constitutes an important problem of public health. Within the feminine group, one of the most frequent cancers is the breast cancer. In Europe esteem that 8 of each 100 women will develop is disease before the 75 years.

Surgery and radiotherapy can cause postsurgical alterations that diminish the quality of life of these patients. With this article it is tried to make a bibliographical search that clarify the postsurgical alterations in this type of patient and the physical therapy treatment used to treat them.

All the analyzed studies indicate the appearance of shoulder alterations in these patients and the benefits that the physical therapy treatment carries to them, no study testifies opposite results.

Although it is certain that any study with the sufficient sample does not exist to clarify wich type of patients (age, physical training conditions, type of surgery, radiotherapy recieved) presents a greater incidence of these alterations.
\end{abstract}

Palabras clave

Fisioterapia; Cáncer de mama; Hombro; Hombro doloroso

Key words

Physical therapy modalities; Breast neoplasms; Shoulder joint; Shoulder pain 


\section{INTRODUCCIÓN}

El cáncer es un conjunto de enfermedades caracteriza- das por la existencia de una proliferación anormal de células. Lo que confiere la característica de malignidad a esta proliferación celular es su capacidad para invadir órganos y tejidos y diseminarse a distancia ${ }^{1}$.

En términos absolutos, el cáncer es la primera causa de muerte en España, con 91.623 muertes en 2000 (57.382 en hombres y 34.241 en mujeres), lo que su- puso el 25,6 \% de todas las defunciones. Esto representa un incremento de 679 defunciones sobre el número de 1999. En ese mismo año, el cáncer pasaba a ser la primera causa de muerte en hombres para el conjunto de España y para las Comunidades Autónomas de Aragón, Asturias, Cantabria, Castilla-León, Galicia, Madrid, Navarra, Vasca y La Rioja. En mujeres, se sitúa en segundo lugar después de las enfermedades cardiovasculares ${ }^{1}$.

El cáncer, que es la causa más frecuente de muerte prematura y evitable en la Unión Europea, constituye un problema importante de salud públical.

El envejecimiento de la población, el incremento de la incidencia de muchos tumores malignos y la mejor su- pervivencia de los enfermos de cáncer debida a los avances diagnósticos y terapéuticos, han supuesto un aumento significativo del número de pacientes de cáncer (prevalencia), situación que pone a prueba la red de asistencia oncológica y que marca la creciente importancia de este grupo de enfermedades como problema de salud pública en nuestro país. Las localizaciones tumorales más frecuentes en España (excluyendo los tumores de piel no-melanoma) son el cáncer de pulmón, los cánceres colorrectales, el cáncer de próstata y el de vejiga en hombres y, en mujeres, el cáncer de mama, los tumores colorrectales, el cáncer de útero, ovario y el de estómago ${ }^{1}$.

El cáncer en Galicia es uno de los principales problemas sanitarios. Constituye la segunda causa de muerte, la primera entre los 35 y 64 años y la segunda en el grupo de 1 a 14 años. La morbilidad es también alta, y ambas son la causa de la gran repercusión que tiene el cáncer en la salud de los ciudadanos de nuestra comunidad. Los recursos que se destinan a su diagnóstico y tratamiento son cada día mayores, y aumentarán previsiblemente en los próximos años, en razón fundamentalmente, al aumento del envejecimiento y mejora de la supervivencia ${ }^{2}$.

La mama está compuesta por lóbulos y conductos. Cada mama tiene 15 a 20 secciones llamadas lóbulos, las cuales comprenden secciones más pequeñas denomina- das lobulillos. Los lobulillos terminan en docenas de bulbos minúsculos que pueden producir leche. Los lóbulos, los lobulillos y los bulbos están conectados por tubos delgados denominados conductos.

El tipo más común de cáncer de mama es el carcinoma ductal, el cual comienza en las células de los con- ductos. El cáncer que se origina en los lóbulos o los 10- bulillos se denomina carcinoma lobular y se encuentra con mayor frecuencia en ambas mamas que otros tipos de cáncer de mama. El cáncer inflamatorio de mama es un tipo de cáncer poco frecuente en el cual la mama está caliente al tacto, enrojecida e inflamada.

El cáncer de mama es el tumor más frecuente en las mujeres occidentales, estimándose que en los países de la Unión Europea, la probabilidad de desarrollar un cáncer de mama antes de los 75 años es del $8 \%$. Anualmente en nuestro país se diagnostican unos 16000 casos al año y produce la muerte de casi 6000 mujeres. En España, la tasa de incidencia ajustada por edad en 1998 era de 67 por 100.000. Se estima que actualmente en España existirían 67.600 mujeres diagnosticadas de cáncer de mama en los últimos 5 años. La mortalidad por cáncer de mama en España comienza a descender en el año 1992, a un ritmo del $2 \%$ anual $^{1}$. Por otro lado, en el Centro Oncológico de Galicia, se registraron 405 casos durante el año $2002^{2}$.

Por su importancia, la investigación, el diagnóstico y el tratamiento del cáncer de mama deben ser aspectos considerados como prioritarios dentro de la política sanitaria. Desde el punto de vista de la prevención secundaria es importante continuar los programas de diagnóstico precoz, evitar los retrasos diagnósticos y asegurar a las pacientes la mejor estrategia terapéutica.

Para poder tratar a este tipo de pacientes, es necesario conocer el estadio en el que se encuentra, para ello, se ha descrito un sistema de clasificación desarrollado por el Comité Estadounidense Conjunto sobre el Cáncer (AJCC, por sus siglas en inglés) que proporciona una estrategia para agrupar los pacientes con respecto a su pro- nóstico. Las decisiones terapéuticas se formulan en parte de acuerdo con las categorías 
del sistema de clasificación, pero principalmente de acuerdo al tamaño del tumor, estado de los ganglios linfáticos, los índices de los receptores de estrógeno y progesterona en el tejido tumoral, el estado menopáusico y la salud general de la paciente.

El AJCC ha designado los estadios mediante la clasificación $\mathrm{TNM}^{3}$. Este sistema se modificó en $2002^{4}$. La clasificación 2002 considera algunas de las categorías nodulares como estadio III las cuales anteriormente se consideraron estadio II. Como resultado del fenómeno de "migración de estadio", la supervivencia por estadio de series de casos clasificados por el nuevo sistema parecerá superior a aquellos que usan el viejo sistema ${ }^{5}$.

\section{MATERIAL Y MÉTODOS}

Viendo la importancia de esta patología en la población, se planifica una búsqueda bibliográfica para conocer la incidencia de complicaciones postquirúrgicas y postradioterápicas susceptibles de tratamiento fisioterápico, prestando especial atención a aquellas más observadas en la unidad de Fisioterapia del Centro Oncoló- gico Regional de Galicia, es decir, aquellas relacionadas con la articulación escápulohumeral.

Para ello se realizar una búsqueda en Medline, Embase, Pascal, Amed, Cinhal, Cochrane y PEDro. Se utilizan las siguientes palabras clave prestando especial atención a los tesauros de Medline y Embase por ser los más completos y utilizados:

"Breast Neoplasms", "Papilloma, Intraductal", "Carcinoma, Lobular", "Breast Self-Examination", "Ultrasonography, Mammary", "Mammary Neoplasms, Experimental", "Carcinoma, Ductal, Breast", "Mastectomy, Segmental" .

"Physical Therapy Modalities", "Physical Therapy (Specialty)", "Physical Therapy Department, Hospital", "Exercise Movement Techniques", "Postoperative Care", "Preoperative Care", "Musculoskeletal Manipulations".

\section{RESULTADOS}

La fisioterapia puede ser enfocada tanto en su vertiente de prevención primaria (numerosos estudios atestiguan que la fisioterapia es un factor protector del cáncer de mama ${ }^{6-16}$ como desde su vertiente terapéutica. Es desde esta donde se concentran un mayor número de investigaciones.

Podríamos dividir, dentro del tratamiento del cáncer de mama, tres grandes líneas de actuación terapéutica:

a) Postoperatorio inmediato, para el tratamiento de las alteraciones secundarias al tratamiento del cáncer.

b) Tratamiento de los efectos secundarios del tratamiento quimioterápico principalmente como puede ser la fatiga, disminución de glóbulos blancos.

c) Tratamiento de las alteraciones iatrogénicas a largo plazo como es el caso del linfedema. El linfedema como consecuencia del manejo del cáncer sigue siendo una preocupación importante para la calidad de vida del paciente con cáncer de mama. El tratamiento de modalidad única de la axila (cirugía o radiación) está relacionado con una baja incidencia de edema del brazo. La radioterapia axilar puede aumentar el riesgo de edema del brazo en las pacientes que se han sometido a disección axilar desde $2 \%$ a $10 \%$ con disección nada más, hasta $13 \%$ a $18 \%$ con radioterapia adyuvante (fig. 1). 


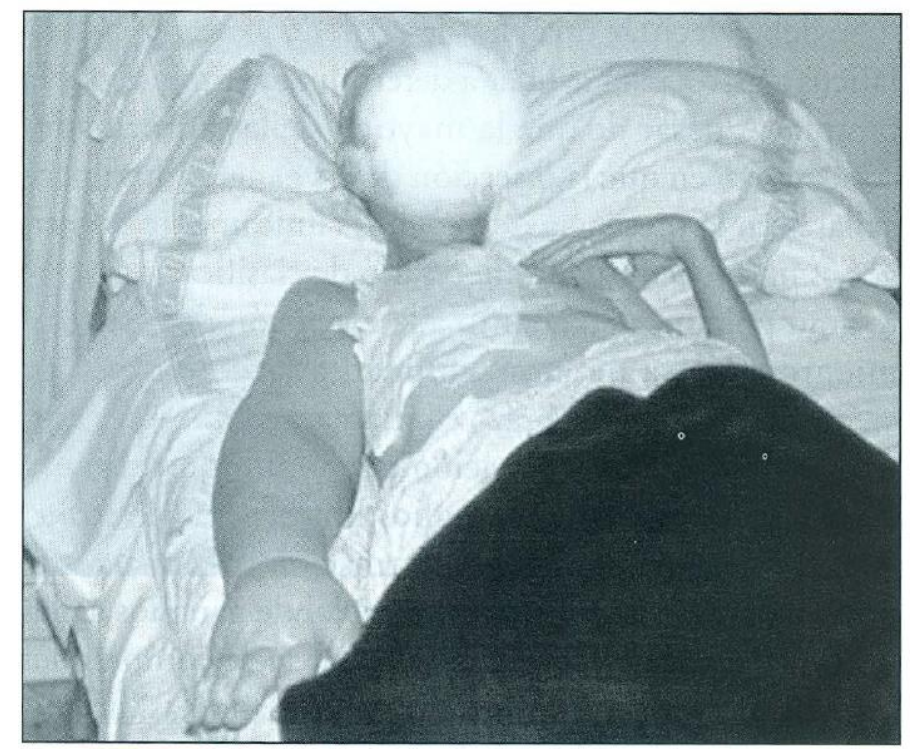

Fig. 1. Linfedema de miembro superior derecho tras intervención de cáncer de mama. El edema apareció tres años después de la cirugía y radioterapia administradas. Obsérvese la diferencia entre ambos miembros.

El objetivo de la presente búsqueda se centra en el primero de los apartados, más concretamente en las alteraciones provocadas por la cirugía y la radioterapia en el complejo glenohumeral.

La cirugía del cáncer de mama presenta varias técnicas posibles. Su elección dependerá del control locoregional del tumor, los nódulos afectados, el pronóstico de la patología y finalmente por los aspectos cosméticos. La ci- rugía se realizará por lo tanto una vez diagnosticado de forma fehaciente tanto de forma clínica, por imagen y en caso de ser necesario biopsia ${ }^{17}$.

En base de los resultados preoperatorios, el procedimiento quirúrgico ha de ser discutido por el equipo multidisciplinar teniendo como objetivo la curación del paciente y tratando de evitar en la medida de lo posible los efectos secundarios de la cirugía ${ }^{17}$.

Las opciones quirúrgicas para tratar el tumor primario incluyen la cirugía preservadora del seno más radioterapia, la mastectomía con reconstrucción y la mastectomía sola (fig. 2). Se debe efectuar una disección de ganglio linfático axilar para fines de clasificación. La supervivencia es equivalente con cualquiera de estas opciones como está documentado en ensayos aleatorios prospectivos ${ }^{18-25}$. La selección del enfoque terapéutico apropiado depende de la ubicación y el tamaño de la lesión, el análisis de la mamografía, el tamaño del seno y la actitud de la paciente acerca de la preservación del seno. La presencia de enfermedad multifocal en el seno o antecedentes de enfermedad vascular colagénica son contraindicaciones relativas a la terapia preservadora del seno ${ }^{26}$. 


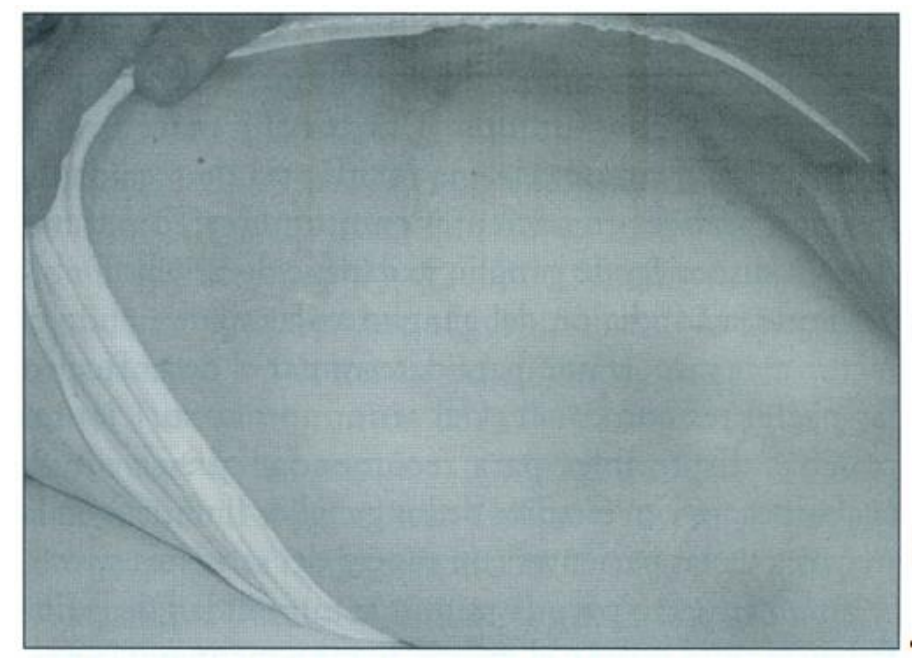

Fig. 2. Mastectomía completa de mama izquierda. La presente paciente presenta una limitación articular a nivel de la articulación glenohumeral de 28 sobre los 40 puntos máximos del test de Constat modificado (valoración de arco articular sin incluir parámetros de fuerza muscular).

La cirugía preservadora del seno sola sin radioterapia ha sido comparada con la cirugía preservadora del seno seguida de radioterapia en seis ensayos aleatorios ${ }^{18,27-32}$. En 2 de estos ensayos, todos los pacientes también recibieron tamoxifeno adyuvante ${ }^{30,31}$. Cada ensayo mostró una tasa general de recurrencia más baja en el seno con la radioterapia, y este efecto estuvo presente en todos los subgrupos de pacientes. En algunos grupos, por ejemplo, las mujeres con tumores pequeños de receptores positivos ${ }^{30}$ y aquellas mayores de 70 años $^{32}$, la reducción absoluta en la tasa de recurrencia fue pequeña $(<5 \%)$. La administración de radioterapia está relacionada con una morbilidad de corto plazo, inconvenientes y complicaciones potenciales a largo plazo $^{32}$.

Se debe efectuar una clasificación de los ganglios linfáticos axilares para ayudar a determinar la prognosis y la terapia. A pesar de que la mayoría de las autoridades concuerdan en que la disección de los ganglios linfáticos axilares en la presencia de ganglios clínicamente negativos es un procedimiento necesario de clasificación, existe gran polémica en cuanto a la extensión del procedimiento a causa de la morbilidad a largo plazo (por ejemplo, molestia del brazo e inflamación) que se le asocia. También hay información que indica que el grado de complicación de los ganglios linfáticos (I contra II contra III) no proporciona información adicional independiente indicadora al número total de ganglios axila- res positivos $^{33}$. La evaluación normal suele comprender solamente disección a nivel I y II, extirpando así un número satisfactorio de ganglio s para su evaluación (por ejemplo, entre 6 y 10 como mínimo) y reduciendo al mismo tiempo la morbilidad del procedimiento. Varios grupos han intentado definir una población de mujeres en quienes las probabilidades de metástasis ganglionar es lo suficientemente baja como para obviar una biopsia de ganglios axilares. En esta serie de casos llevada a cabo en una sola institución, la incidencia de ganglios positivos en pacientes con tumores T1a, osciló entre $9 \%$ y $16 \%{ }^{33,34}$. En otras series, la incidencia de recaída de ganglios axilares en pacientes con tumores T1a, tratados sin disección de ganglio axilar fue de $2 \%^{35}$. Debido a que la condición del ganglio axilar sigue siendo el factor más importante para determinar el desenlace en las pacientes con cáncer del seno, no hay suficientes pruebas disponibles para recomendar que se omita la clasificación en estadios de los ganglios linfáticos en la mayoría de las pacientes con cáncer del seno invasivo.

En un esfuerzo para disminuir la morbilidad de la linfadenectomía axilar y al mismo tiempo mantener la precisión de la clasificación, varios investigadores han estudiado la cartografía linfática y la biopsia del ganglio linfático centinela (SLN, por sus siglas en inglés) en mujeres con cáncer infiltran te del seno ${ }^{36-39}$. El SLN se define como el primer ganglio en la cuenca linfática que recibe flujo linfático primario. Las investigaciones han mostrado que una inyección de azufre coloidal radioactivado con tecnecio solo, con colorante azul vital o ambos en torno al tumor o la cavidad de la biopsia o en la región subareolar, y el drenaje posterior de estos compuestos a la axila puede identificar el SLN en $92 \%$ a $98 \%$ de las pacientes $^{40,41}$. Estos informes muestran una concordancia de 97,5\% a $100 \%$ entre la biopsia del SLN y la disección completa de ganglios linfáticos axilares ${ }^{36-39}$. Los resultados de un ensayo aleatorio con 532 
pacientes con carcinomas T1 sometidos a biopsia SLN más disección axilar completa o biopsia SLN sola, no mostró recurrencias axilares en ninguno de los grupos ni diferencia alguna en la supervivencia sin enfermedad durante tres años ${ }^{42}$.

La tasa falsa negativa observada (como el número de pacientes con biopsia del SLN negativa dividido por el número de pacientes con ganglios axilares positivos en el momento de la disección de ganglios axilares) de la biopsia SLN varía entre $0 \%$ y $10 \%$. La tasa de éxito depende de la experiencia del cirujano y las características del tumor primario. Por lo general, los estudios han limitado el uso de la biopsia del SLN a las mujeres con enfermedad T1 y T2 que no presenten complicación multifocal ni ganglios linfáticos clínicamente positivos. La biopsia SLN sola está relacionada con menor morbilidad que la linfadenoctomía axilar. Los ensayos clínicos aleatorios en curso, ayudarán a determinar si ambos procedimientos rinden tasas de supervivencia comparables y si existe algún beneficio terapéutico al llevar a cabo una linfadenoctomía axilar completa en los pacientes con metástasis SLN.

\section{Alteración de la articulación glenohumeral}

Fruto del tratamiento descrito hasta ahora, numerosos estudios refieren las complicaciones que aparecen en la articulación glenohumeral, así Bochdansky, realizó un estudio en que dividió a 119 pacientes en cuatro grupos, uno de sujetos sanos, uno de pacientes tras mastectomía radical modificada, el tercero con la misma intervención y reconstrucción de mama con el músculo dorsal largo y el cuarto con la misma intervención y reconstrucción de mama con reconstrucción microvascular del dorsal largo. Estudio el rango de movimiento, la mobilidad, test de destreza y fuerza muscular isométrica y observó una disminución del movimiento y la fuerza muscular en los tres grupos operados ${ }^{43}$.

Box ${ }^{44}$, investigó dos grupos de pacientes sometidas a cirugía conservadora en cáncer de mama, aleatorizó a 65 mujeres en dos grupos, un grupo de tratamiento de fisioterapia y un grupo control que recibió consejos sobre ejercicios adecuados para su hombro. Observó que el grupo experimental presentaba una abducción correcta más rápidamente que el grupo control. La recuperación funcional de la articulación glenohumeral también aumentó en el grupo experimental.

Karki $^{45}$ examinó de forma retrospectiva a 105 pacientes 6 meses después de su operación para evaluar sus instrucciones de realización de ejercicios a estos pacientes. Evaluó la movilidad de la articulación, la fuerza del miembro superior y su uso en las actividades de la vida diaria. Todos sus pacientes demandaban en este estudio un programa de ejercicios que les capacitase para paliar estas limitaciones.

Lauridsen $^{46}$ señaló en un estudio realizado en el 2000 que muchas mujeres experimentan síntomas tardíos como consecuencia del tratamiento quirúrgico del cáncer de mama como una disminución en la fuerza del miembro superior afectado, reducción del arco de movimiento y aumento del tono muscular. También observó que el tratamiento de fisioterapia podía tratar estos síntomas tardíos e incluso conseguir su no aparición. En un estudio posterior ${ }^{47}$ comprobó que las limitaciones articulares glenohumerales eran mayores en las pacientes sometidas a mastectomía radical modificada que en aquellas pacientes sometidas a tratamiento conservador de la mama.

Según Morimoto, no existen diferencias entre los pacientes que reciben mastectomía de los pacientes con terapia conservadora de la mama, salvo en la abducción del hombro a la cuarta semana de la operación, por otro lado, el $15 \%$ de los pacientes presentaba dolor después de 12 semanas ${ }^{48}$.

Tengrup, en un estudio realizado sobre 110 pacientes, observó un $49 \%$ de pacientes con problemas de movilidad del miembro superior, siendo más precisos, el $57 \%$ de los que habían recibido radioterapia postquirúrgica (fig. 3) y el $30 \%$ de los que no habían recibido este tratamiento, y casi un $20 \%$ desarrollaron un linfedema ${ }^{49}$. 


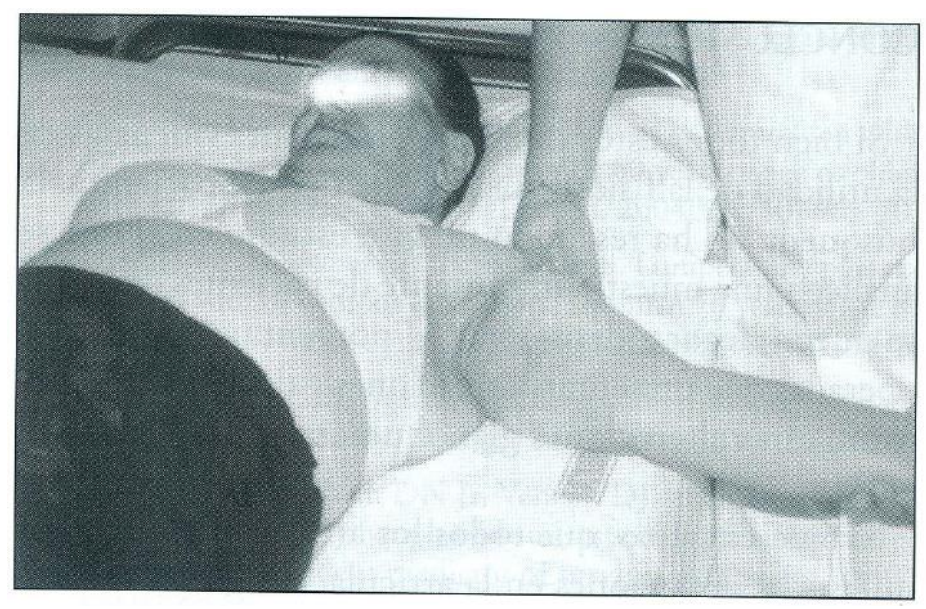

Fig. 3. Paciente operada de cáncer de mama a tratamiento de radioterapia. Obsérvese la cicatriz quirúrgica a nivel axilar debido a la exéresis de los nódulos linfáticos.

Según Le et al $^{50}$, ya en el año 1997, y fruto de la comparación de cuatro grupos de pacientes, uno con tratamiento de fisioterapia, otro con movilización de hombro, otro con los dos y otro con ninguno, demostraron que el rango de movimiento era mayor en el grupo que unía fisioterapia y movilización, que el dolor era menor en los grupos que incluían las movilizaciones y que el volumen de seroma drenado era menor en los grupos que incluían fisioterapia.

Iohansson $^{51}$ en el 2001 analizó durante dos años a un conjunto de 61 mujeres que sufrieron disección axilar con y sin radioterapia y descubrió una disminución del arco de movimiento después de un mes de la operación, un aumento del volumen del miembro después de dos meses y una disminución de la fuerza muscular después de 6 meses de la operación.

Siguiendo la misma línea, Collins ${ }^{52}$ investigó la vuelta al trabajo de las mujeres operadas de cáncer de mama y observó que llevaba más tiempo que lo aceptado como normal en la sociedad científica, las principales causas de este retardo estaban relacionadas con problemas glenohumerales al dormir, disminución de la capacidad de hacer el trabajo del hogar, arreglar el jardín y dolor. Y Hincan ${ }^{53}$ en su muestra asegura que el $20 \%$ de las mujeres investigadas, no habían vuelto a trabajar, y que de ellas, el $50 \%$ no había recibido ningún tratamiento de fisioterapia.

Por ultimo Box ha demostrado, que un trabajo fisioterápico postquirúrgico puede reducir la aparición de linfedemas futuros ${ }^{54}$.

\section{CONCLUSIONES}

Si bien todos estos estudios, presentan evidencias de la utilidad de la fisioterapia en este tipo de pacientes, la búsqueda no ha resaltado ningún estudio en el que se analicen una muestra importante de pacientes en la que se pueda llegar a demostrar, el porcentaje de mujeres operadas que presentan alteraciones y debido a que factores, estas alteraciones glenohumerales se ven favorecidas.

Si bien es cierto, que todos los artículos consultados muestran alteraciones en la articulación glenohumeral de estas pacientes, y ningún artículo refiere una serie de mujeres operadas de cáncer de mama y que no presen- ten esta alteración.

Por otro lado, la experiencia clínica nos indica que es- tas alteraciones son claras y debidas en todos los casos al tratamiento previo recibido. 
Por lo tanto se hace necesario la realización de un estudio dirigido a conocer de forma clara, cuales son las características comunes que presentan estos pacientes para poder determinar cuales serán susceptibles de recibir tratamiento fisioterápico precoz y e incluso trata- miento preoperarorio.

En la mayoría de las ocasiones, estos pacientes reciben consejos sobre como realizar diversos ejercicios en casa, consejos que no forman parte de un protocolo de tratamiento propuesto por un fisioterapeuta. Finalmente estas pacientes acuden a un servicio de fisioterapia para solucionar su problema.

\section{BIBLIOGRAFÍA}

1. López-Abente G, Pollán M, Aragonés N, Pérez-Gómez B. Informe sobre la salud de los españoles. Cáncer. Instituto de Salud Carlos III. Madrid; 2003. p. 1-3.

2. Pira López MJ, Candal Seijas JL, López Novio MJ. Registro Hospitalario de Tumores de! Centro Oncológico de Galicia, año 2002. Centro Oncológico Regional de Galicia. La Coruña; 2002. p. 5-33.

3. Breast. In: American Joint Committee on Cancer: AJCC Cancer Staging Manual. 6th ed. New York, NY: Springer; 2002. p.171-80.

4. Singlerary SE, Allred C, Ashley P, et al. Revision of the American Joint Commmittee on Cancer Staging system for breast cancer. J Clin Oncol. 2002;20(17):3628-36.

5. Woodward WA, Strom EA, Tucker SL, et al. Changes in the 2003 American Joint Committee on Cancer Staging for breast cancer dramatically affect stage-specific survival. J Clin Oncol. 2003;21 (17):3244-8.

6. Margolis KL, Mucci L, Braaten T, Kumle M, Trolle LY, Adami HO, et al. Physical activity in different periods of life and the risk of breast cancer: the Norwegian-Swedish Women's Lifestyle and Health cohort study. Cancer Epidemiol Biomarkers Prev. 2005; 14(1):27 -32.

7. Chlebowski RT, Pettinger M, Stefanick ML, Howard BY, Mossavar-Rahmani Y, McTiernan A. Insulin, physical activity, and caloric intake in postmenopausal women: breast cancer implications. J Clin Oncol. 2004;22(22):4507-13.

8. Chlebowski RT, Pettinger M, Stefanick ML, Howard BY, Mossavar-Rahmani Y, McTiernan A. Insulin, physical activity, and caloric intake in postmenopausal women: breast cancer implications. J Clin Oncol. 2004;22(22):4507-13.

9. Matthews CE, Fowke JH, Dai Q, Leon BH, Jin F, Shu XO, et al. Physical activity, body size, and estrogen metabolism in women. Cancer Causes Control. 2004; 15(5):473-81.

10. McTiernan A, Tworoger SS, Rajan KB, Yasui Y, Sorenson B, Ulrich CM, et al. Effect of exercise on serum androgens in postmenopausal women: a 12-month randomized clinical trial, Cancer Epidemiol Biomarkers Prev. 2004;13(7):1099-105.

11. McTiernan A, Tworoger SS, Rajan KB, Yasui Y, Sorenson B, Ulrich CM, et al. Effect of exercise on serum androgens in postmenopausal women: a 12-month randomized clinical trial, Cancer Epidemiol Biomarkers Prev. 2004;13(7):1099-105.

12. Chlebowski RT. Reducing the risk of breast cancer. New England Journal of Medicine. 2000;343(3): 191-8.

13. Culos-Reed SN. Physical activity and cancer in youth: A review of physical activity's protective and rehabilitative functions. Pediatric Exercise Science. 2002;14(3):248-58.

14. Demark WW, Clipp EC, Morey MC, Pieper CF, Sloane R, Snyder DC, et al. Physical function and associations with diet and exercise: Results of a cross-sectional survey among elders with breast or prostate cancer. International Journal of Behavioral Nutrition and Physical Activity, 2004; $1: 6$.

15. Rintala PE. Low intensity exercise in healthy-weight women reduces risk of breast cancer. Evidence Based Healthcare. 2004; 8(2):69-70.

16. Salih AK, Fentiman IS. 14. Breast cancer prevention. International Journal of Clinical Practice, 2002;56(4):26771.

17. Rutgers EJ. Guidelines to assure quality in breast cancer surgery. Eur J Surg Oncol. 2005;31(6):568-76.

18. Fisher B, Anderson S, Bryant J, et al. Twenty-year follow-up of a randomized trial comparing total mastectomy, lumpectomy, and lumpectomy plus irradiation for the treatment of invasive breast cancer. N Engl J Med. 2002;347(16):1233-41.

19. Blichert-Toft M, Rose C, Andersen JA, et al. Danish randomized trial comparing breast conservation therapy with mastectomy: six years of life-table analysis. Danish Breast Cancer Cooperative Group. J Natl Cancer Inst Monogr. 1992;(11):19-25.

20. Van Dongen JA, Bartelink H, Fentiman IS, et al. Randomized clinical trial to assess the value of breastconserving therapy in stage I and II breast cancer, EORTC 10801 trial. J Natl Cancer Inst Monogr. 1992;(11):158.

21. Sarrazin D, Le MG, Arriagada R, et al. Ten-year results of a randomized trial comparing a conservative treatment to mastectomy in early breast cancer. Radiother Oncol. 1989;14: 177-84.

22. Jacobson JA, Danforth DN, Cowan KH, et al. Ten-year results of a comparison of conservation with mastectomy in the treatment of stage I and II breast cancer. N Engl J Med. 1995;332 (14):907-11.

23. Veronesi U, Cascinelli N, Mariani L, et al. Twenty-year follow-up of a randomized study comparing breastconserving surgery with radical mastectomy for early breast cancer. N Engl J Med. 2002;347(16):1227-32.

24. Veronesi U, Salvadori B, Luini A, et al. Breast conservation is a safe method in patients with small cancer of the breast. Long-term results of three randomised trials on 1,973 patients. Eur J Cancer. 1995;31A(10):1574-9. 
25. Van Dongen JA, Yoogd AC, Fentiman IS, et al. Long-term results of a randomized trial comparing breastconserving therapy with mastectomy: European Organization for Research and Treatrnent of Cancer 10801 trial. J Natl Cancer Inst, 2002;92 (14):1143-50.

26. Abrams JS, Phillips PH, Friedman MA. Meeting highlights: a reappraisal of research results for the local treatment of early stage breast cancer. J Natl Cancer Inst, 1995;87(24):1837-45.

27. Veronesi U, Luini A, Del Vecchio M, et al. Radiotherapy after breast-preserving surgery in women with localized cancer of the breast. N Engl J Med. 1993;328(22):1587-91.

28. Liljegren G, Holmberg L, Bergh J, et al. 10-Year results alter sector resection with or without postoperative radiotherapy for stage I breast cancer: a randomized trial. J Clin Oncol. 1999; 17(8):2326-33.

29. Clark RM, McCulloch PB, Levine MN, et al. Randomized clinical trial to assess the effectiveness of breast irradiation following lumpectomy and axillary dissection for node-negative breast cancer. J Natl Cancer Inst. 1992;84(9):683-9.

30. Fyles AW, McCready DR, Manchul LA, et al. Tamoxifen with or without breast irradiation in women 50 years of age or older with early breast cancer. N Engl J Med. 2004;351 (10):963-70.

31. Hughes KS, Schnaper LA, Berry D, et al. Lumpectomy plus tamoxifen with or without irradiation in women 70 years of age or older with early breast cancer. N Engl J Med. 2004;351(10):971-7.

32. Smith IE, Ross GM. Breast radiotherapy after lumpectomy-no longer always necessary. N Engl J Med. 2004;351 (10): 1021-3.

33. Barth RJ Jr, Danforth DN Jr, Venzan DJ, et al. Level of axillary involvement by lymph node metastases from breast cancer is not an independent predictor of survival. Arch Surg. 1991; 126(5):574-7.

34. Rivadeneira DE, Simmons RM, Christos PJ, et al. Predictive factors associated with axillary lymph node metastases in T1a and T1b breast carcinomas: analysis in more than 900 patients. J Am Coll Surg. 2000;191(1):16; discussion 6-8.

35. Greco M, Agresti R, Cascinelli N, et al. Breast cancer patients treated without axillary surgery: clinical implications and biologic analysis. Ann Surg. 2000;232(1):1-7.

36. Veronesi U, Paganelli G, Galimberti Y, et al. Sentinel-node biopsy to avoid axillary dissection in breast cancer with clinically negative lymph-nodes, Lancet. 1997;349(9069):1864-7.

37. Albertini JJ, Lyman GH, Cox C, et al. Lymphatic mapping and sentinel no de biopsy in the patient with breast cancer. JAMA. 1996;276(22):1818-22.

38. Krag D, Weaver D, Ashikaga T, et al. The sentinel node in breast cancer-a multicenter validation study. N Engl J Med. 1998; 339(14):941-6.

39. Veronesi U, Paganelli G, Viale G, et al. Sentinel lymph node biopsy and axillary dissection in breast cancer: results in a large series. J Natl Cancer Inst, 1999;91 (4):368-73.

40. Kern KA. Sentinel lymph node mapping in breast cancer using subareolar injection of blue dye. J Am Coll Surg. 1999;189(6):539-45.

41. Rubio IT, Korourian S, Cowan C, et al. Sentinel lymph node biopsy for staging breast cancer. Am J Surg. 1998;176(6):532-7.

42. Veronesi U, Paganelli G, Yiale G, et al. A randomized comparison of sentinel-node biopsy with routine axillary dissection in breast cancer. N Engl J Med. 2003;349(6):546-53.

43. BochdanskyT, Zauner DA, Alacamlioglu Y, Schemper M, Piza KH. Evaluation of shoulder functian subsequent to mastectomy with and without transfer of the latissimus dorsi muscle, and after microvascular transfer of the latissimus dorsi muscle. Acta Chirurgica Austriaca. 1995;(2): 104-8.

44. Box RC, Reul-Hirche HM, Bullock-Saxton JE, Furnival CM. Shoulder movement after breast cancer surgery: results of a randomised controlled study of postoperative physiotherapy. Breast Cancer Res Treat. 2002;75(1):3550 .

45. Karki A, Simonen R, Malkia E, Selfe J. Postoperarive education concerning the use of the upper limb., and exercise and treatment of the upper limb: cross-sectional survey of 105 breast cancer patients. Support Care Cancer, 2004;12(5):347-54.

46. Lauridsen MC, Torsleff KR, Husted H, Erichsen C. Physiotherapy treatment of late symptoms following surgical treatment of breast cancer. Breast. 2000;9(1):45-51.

47. Lauridsen MC, Christiansen p, Hessov I. The effect of physiotherapy on shoulder function in patients surgically treated for breast cancer: A randomized study. Acta Oncol. 2005;44(5):449-57.

48. Morimoto T, Tamura A, Ichihara T, Minakawa T, Kuwamura Y, Miki Y, et al. Evaluation of a new rehabilitation program for postoperative patients with breast cancer. Nurs Health Sci. 2003;5(4):275-82.

49. Tengrup I, Tennvall NL, Christiansson I, Laurin M. Arm morbidity after breast-conserving therapy for breast cancer. Acta Oncologica.2000;39(3):393-7.

50. Le VB, Dumorrier A, Guillaume MY, Mouriesse H, Barreau PL. Physiotherapy after surgery for breast cancer. Bulletin du Cancer. 1997;(10):957 -61.

51. Johansson K, Ingvar C, Albertsson M, Ekdahl C. Arm lymphoedema, shoulder mobility and muscle strength after breast cancer treatment - A prospective 2-year study. Advances in Physiotherapy. 2001;3(2):55-66.

52. Collins LG, Nash R, Round T, Newman B. Perceptions of up- per-body problems during recovery from breast cancer treatment. Support Care Cancer. 2004;12(2):106-13.

53. Hinman MR. Factors influencing work disability for women who have undergone mastectomy. Women and Health. 2001; 34(2):45-60.

54. Box RC, Reul-Hirche HM, Bullock-Saxcon JE, Furnival CM. Physiotherapy after breast cancer surgery: Results of a randomised controlled study to minimise lymphoedema. Breast Cancer Research and Treatment. 2002;75(1):51-64. 